\title{
SOME CHARACTERIZATIONS OF SEMI-LOCALLY CONNECTED SPACES
}

\author{
YU-LEE LEE ${ }^{1}$
}

Let $(X, \mathfrak{u})$ be a connected $T_{1}$-space and let $\mathfrak{e}$ be the class of all closed connected subsets of $(X, \mathcal{u})$. Define the operator $K$ on all the subsets of $X$ as follows: $K(A)$ is the intersection of all the finite unions of elements of $\mathfrak{C}$ which cover $A$. Then we see that $K$ has the following properties:

(1) If $A \subset X$, then $K(A)$ is closed and $\mathrm{Cl}(A) \subset K(A)$.

(2) If $A$ is connected in (X, $\mathfrak{u})$, then $\mathrm{Cl}(A)=K(A)$.

(3) If $A$ is closed and connected, then $K(A)=A$.

(4) The operator $K$ satisfies the Kuratowski axioms.

Thus $K$ defines a new topology $v$ for $X$, we call $v$ the derived topology of น.

(5) The space $(X, \vartheta)$ is a connected $T_{1}$-space.

(6) If $A$ is connected in $(X, \mathcal{u})$, then $A$ is connected in $(X, v)$.

(7) If $A$ is closed and connected in $(X, \mathcal{u})$, then $A$ is closed and connected in $(X, v)$.

(8) The topology $v$ is contained in $u$.

Recall from [1] that a connected $T_{1}$-space $X$ is said to be semilocally connected (s.l.c.) at $x$ provided there exists a local open base at $x$ such that $X-V$ has only a finite number of components for any $V$ in the local open base at $x$. The space $X$ is s.l.c. provided $X$ is s.l.c. at every $x$ in $X$.

Theorem 1. The connected $T_{1}$-space $(X, \mathcal{U})$ is s.l.c. if and only if $\mathrm{Cl}(A)=K(A)$ for any subsets $A$ of $X$.

Proof. Suppose $(X, \mathfrak{u})$ is s.l.c., $A \subset X$ and $x \notin \mathrm{Cl}(A)$. Then there exists an open neighborhood $V$ of $x$ such that $X-V$ is the union of a finite number of closed connected sets which cover $A$. Hence $x \in K(A)$ and therefore $\mathrm{Cl}(A)=K(A)$.

Conversely suppose $\mathrm{Cl}(A)=K(A)$ for all $A \subset X$ and $U$ is an open neighborhood of a point $x$ in $X$. Since $\mathrm{Cl}(X-U)=K(X-U)$, we have $x \notin K(X-U)$. Therefore there exists a finite family of closed connected sets $C_{1}, C_{2}, \cdots, C_{n}$ which covers $X-U$ and such that $x \notin C_{i}$ for each $i=1,2, \cdots, n$. Since $X-U\left\{C_{i}: i=1,2, \cdots, n\right\}$ is open and is contained in $U$, it follows that $X$ is s.l.c. at $x$.

Received by the editors October 7, 1964.

1 This research was supported by the National Science Foundation under Grant number GP-1457. 
Theorem 2. Let $(X, \mathcal{u})$ be a connected $T_{1}$-space and let $v$ be the derived topology of u. Then $(X, v)$ is s.l.c.

Proof. Since $(X, V)$ is a connected $T_{1}$-space by (5), we can consider the derived topology $W$ of $V$ with the defining operator $J$. Let $\mathcal{C}$ and $\mathfrak{e}^{\prime}$ be the families of all closed connected subsets of $(X, \mathcal{U})$ and $(X, v)$ respectively. By (1), $\mathrm{Cl}(A) \subset K(A) \subset J(A)$ for any $A \subset X$. Since by (7), $\mathfrak{e} \subset \mathfrak{C}^{\prime}$, it follows that any finite cover of $A$ by elements of $\mathfrak{e}$ is a finite cover of $A$ by elements of $\mathfrak{e}^{\prime}$. Hence by definition of $K$ and $J$ we have $K(A) \supset J(A)$. By Theorem 1, $(X, v)$ is s.l.c.

Corollary 1. A topological space $(X, V)$ is s.l.c. if and only if $v$ is the derived topology of some connected $T_{1}$-space $(X, \mathcal{u})$.

Corollary 2. A locally connected continuum $(X, \mathfrak{u})$ ) is s.l.c.

Proof. Let $A$ be a closed set in $(X, \mathfrak{u})$ and $p \notin A$. Then there exists a finite family of closed connected sets which covers $A$ but not $p$. Hence $p \notin K(A)$ and $K(A)=A$. By Theorem $1,(X, \mathfrak{u})$ is s.l.c.

A mapping $f$ of $(X, \mathcal{U})$ into $(Y, \mathcal{V})$ is called semi-connected if whenever $A$ is a closed connected set in $(Y, v), f^{-1}(A)$ is a closed connected set in $(X, \mathfrak{u})$. The following theorem is a generalization of a result of Tanaka [2] and W. J. Pervin and N. Levine [3].

Theorem 3. A semi-connected mapping $f$ from $(X, \mathcal{u})$ into a s.l.c. space $(Y, V)$ is continuous.

Proof. Let $A$ be a closed subset of $Y$; then

$$
\begin{aligned}
A & =\mathrm{Cl}(A)=K(A) \\
& =\bigcap\left\{\cup\left\{C: C \in \mathbb{e}^{\prime}\right\}\right\}
\end{aligned}
$$

where $\mathfrak{e}^{\prime}$ is a finite family of closed connected subsets which covers $A$. Hence

$$
f^{-1}(A)=\bigcap\left\{\cup\left\{f^{-1}(C): C \in \mathfrak{e}^{\prime}\right\}\right\}
$$

is closed and $f$ is continuous.

Theorem 4. If $(X, \mathcal{u})$ is a connected $T_{1}$-space, then the following statements are equivalent:

(a) $(X, \mathfrak{u})$ is s.l.c.

(b) Every semi-connected mapping from a topological space $Y$ into $(X, \mathfrak{u})$ is continuous.

(c) The identity mapping from $(X, V)$ onto $(X, \mathcal{u})$ is continuous where $v$ is the derived topology of $u$.

Proof. The previous theorem shows that (a) implies (b). The 
identity mapping $i$ is semi-connected, by (7). Hence (b) implies (c). If (c) holds then $i$ is a homeomorphism. By Theorem 2, (X, $u$ ) is s.l.c.

\section{BIBLIOGRAPHY}

1. G. T. Whyburn, Analytic topology, Amer. Math. Soc. Colloq. Publ. Vol. 28, Amer. Math. Soc., Providence, R. I., 1942.

2. T. Tanaka, On the family of connected subsets and the topology of space., J. Math. Soc. Japan 7 (1955), 389-393.

3. W. J. Pervin and N. Levine, Connected mappings of Hausdorff space, Proc. Amer. Math. Soc. 11 (1960), 688-691.

UNIVERSITY OF CONNECTICUT AND UNIVERSITY OF OREGON

\section{A TECHNIQUE FOR CONSTRUCTING EXAMPLES}

\section{MARY ELLEN RUDIN}

The word space in this paper will refer to Hausdorff spaces.

I have recently been asked the following questions.

1 (by the topology class of R. H. Bing). Is there a regular, sequentially compact space in which some nested sequence of continua intersect in a disconnected set?

2 (by E. Michael). Is there a normal, sequentially compact but not compact, space having a separable, metric, locally compact, dense subset?

Examples showing that the answer to both questions is yes, modulo the continuum hypothesis, are easily constructed using a technique I have of ten used before. The technique, described in $\S I$, is perhaps more interesting than the particular examples which are given in \$II. §III gives a variation of the technique and raises some questions.

I. This technique is useful in the construction of pathological spaces having nice dense subsets.

Let $R$ be the wedge in the plane consisting of all points $(x, y)$ such that $0 \leqq x \leqq 1$ and $0 \leqq y \leqq x$; let $T=R-\{(0,0)\}$.

Let $F$ be the set of all continuous real valued functions whose domain is the set of all positive numbers less than or equal to 1 and whose graph lies in $T$.

There is a natural partial ordering of the terms of $F$ : if $f$ and $g$

Received by the editors December 16, 1964. 